\title{
Regional Distribution and Alterations of Lectin Binding to Colorectal Mucin in Mucosal Biopsies from Controls and Subjects with Inflammatory Bowel Disease
}

\author{
Lucien R. Jacobs and P. Wendy Huber \\ Department of Medicine, School of Medicine, University of California, Davis, California 95616
}

\begin{abstract}
Glycoconjugate composition of colorectal goblet cell mucin was characterized according to the anatomical distribution of lectinbinding sites in mucosal biopsies from 35 control subjects and 55 patients with inflammatory bowel disease. 24 of the controls had mucosal inflammation on biopsy, without clinical evidence of inflammatory bowel disease. These inflamed controls showed a similar rate of presence of lectin-binding sites as the normal noninflamed group. In the controls, the frequency of binding of Ricinus communis agglutinin I to galactosyl residues was consistently higher than that found with either Ulex europaeus agglutinin I to fucosyl or Dolichus biflorus agglutinin to $\mathrm{N}$ acetyl galactosyl groups. A significant proximal to distal gradient for Ulex europaeus agglutinin I binding sites was identified in the control group. These binding sites were present four times more often in the proximal colon than in the distal colon ( $P$ $<0.025)$. In the ulcerative and Crohn's colitis groups, this gradient effect was lost, predominantly as a result of decreased availability of fucosyl residues in the proximal colon. In the descending colon of Crohn's colitis tissues, there was a complete absence of Dolichus biflorus agglutinin binding sites compared with the $62.5 \%$ incidence in the control group $(P<0.05)$. These results demonstrate that the expression of lectin-binding sites in human large intestinal goblet mucin is specifically altered in inflammatory bowel disease, indicating that there are changes in glycosylation of colorectal mucin consistent with alterations in goblet cell differentiation.
\end{abstract}

\section{Introduction}

Characterization of the biochemical abnormalities in the colonic mucosa of patients with inflammatory bowel disease has been attempted using a number of different techniques. Histochemical studies of colorectal mucin have demonstrated a decrease in goblet cell mucin in ulcerative colitis, but no specific qualitative difference between ulcerative colitis, Crohn's colitis, and normal patients $(1,2)$. Although increased sialomucin staining occurs when dysplastic changes are present (3), this does not seem to be clinically significant, since nonspecific increases in sialomucins, sulphomucins, and neutral muco-

This work was presented in part at the 84th annual meeting of the American Gastroenterological Association, May 1983, Washington, DC, and appeared in abstract form (1983. Gastroenterology. 84:1406). Address reprint requests to Dr. Jacobs, Division of Gastroenterology, TB 192, University of California, Davis, CA 95616.

Received for publication 23 January 1984 and in revised form 11 September 1984.

J. Clin. Invest.

(c) The American Society for Clinical Investigation, Inc.

$0021-9738 / 85 / 01 / 0112 / 07 \quad \$ 1.00$

Volume 75, January 1985, 112-118 substances may all be found in inflammatory bowel disease (4). The increase in superficial goblet cells, along with a higher percentage containing neutral mucin in the sigmoid region of ulcerative colitis patients, is thought to result from the faster cell turnover rate (5) known to occur in this disease $(6,7)$. However, in severe cases of ulcerative colitis, goblet cells with neutral mucin are decreased or totally absent (5).

Another approach involves the biochemical characterization of colonic mucosal glycoproteins, which form a major constituent of intestinal mucin (8). Measurements of enzymes important in intestinal glycoprotein metabolism have not shown any differences in glycosyltransferase activity in patients with inflammatory bowel disease (9), whereas glucosamine synthetase, a rate-limiting enzyme in glycoprotein synthesis, is increased in normal-appearing rectal mucosa from patients with Crohn's Disease (10). Analyses of human colonic mucus glycoproteins revealed significant reductions in both the carbohydrate content and size of the oligosaccharide units in granulomatous and ulcerative colitis (11). Furthermore, there is also a specific increase in the mannose-containing glycoprotein of colonic mucus in ulcerative colitis (12). More recently, a comparison of partially purified colonic mucin from patients with inflammatory bowel and control patients identified six distinct mucin species. In ulcerative colitis, there was a selective reduction of species IV from sigmoid and ascending colon when compared with control or Crohn's Disease patients (13).

Human mucin glycoconjugate composition also has been examined using lectins (14). These are agglutinins that bind to specific terminal carbohydrates of glycoconjugates and have been used as specific probes to examine the structural and secretory components of human small (15) and large intestine $(16,17)$. A preliminary report of lectin binding to rectal biopsies from ulcerative colitis patients stated that $28 \%$ of samples showed decreased binding with the lectin Dolichus biflorus agglutinin (DBA), ${ }^{1}$ whereas with Ulex europaeus agglutinin I (UEA $)_{1}$ and Ricinus communis agglutinin I (RCA $\mathbf{R}_{1}$, the binding was unchanged from control patients (18). Furthermore, in ulcerative colitis patients developing colon cancer, DBA staining was decreased, while supranuclear binding of peanut agglutinin became pronounced (18). More recent evidence suggests the existence of regional differences in glycoconjugate differentiation, as demonstrated by the uniform presence of $\mathrm{UEA}_{1}$ binding sites in the right colon, in contrast to the left side where tissues are only occasionally positive, or, as in the rectum, are even devoid of UEA $_{1}$ binding sites (17, 19). In contrast, patients with adenomatous polyps or colorectal cancer show $\mathrm{UEA}_{1}$ staining of the tumor (19).

To further localize and characterize large bowel mucosal

1. Abbreviations used in this paper: DBA, Dolichus biflorus agglutinin; FITC, fluorescein isothiocyanate; Fuc, $\alpha$-L-fucose; Gal, D-galactose; GalNAc, $N$-acetyl galactosamine; PAS, periodic acid Schiff; $\mathbf{R C A}_{1}$, Ricinus communis agglutinin I; UEA $\mathrm{U}_{1}$, Ulex europaeus agglutinin I. 
lectin-binding sites, biopsies from all regions of the large intestine were examined. Tissue samples from a control group of subjects, comprising inflamed mucosal biopsies due to conditions other than inflammatory bowel and normal noninflamed samples, were compared with the results found in mucosal biopsies from patients diagnosed as having either ulcerative or Crohn's colitis.

\section{Methods}

Study population. The clinical and histological material in this study was selected retrospectively. The majority of patients had been seen by the gastroenterology service at the University of California, Davis Medical Center, during the preceding 10-yr period. Additional clinical material was provided by other members of the faculty who had personally managed a number of patients with inflammatory bowel disease over several years. The clinical data are summarized in Table I. Subjects composing the control group had been investigated for large bowel disease, and had been found not to have inflammatory bowel disease. Patients with colonic carcinoma and/or polyposis were excluded, since previous studies indicate that intestinal mucin glycoconjugates may be altered in these conditions $(19,20)$. During these investigations, large bowel mucosal biopsies had been performed. According to prior histopathological evaluation, 11 of the 35 control biopsies were morphologically normal, while in the remaining 24 patients there was histological evidence of inflammation. 20 patients in the control group had evidence of mucosal inflammation by proctosigmoidoscopy. However, it was determined by clinical followup, as well as by endoscopic, radiological, and histological criteria, that none of these patients had ulcerative or Crohn's colitis. Among the clinical disorders constituting the control group were four patients with infective and noninfective forms of diarrhea, three with radiation damage, three with ischemia, seven with functional bowel disease, two with colonic pseudo-obstruction, three with colonic diverticular disease, four with infectious proctocolitis, and one with antibiotic-associated colitis. Eight more patients were included with self-limited colitis and/or proctitis, of brief duration and unknown etiology, which did not recur.

Patients were diagnosed as having inflammatory bowel disease according to a typical clinical history, abnormal barium studies, and colonoscopy or proctosigmoidoscopy with biopsies, showing an ap-

Table I. Characteristics of Patient Population

\begin{tabular}{|c|c|c|c|}
\hline & Controls & $\begin{array}{l}\text { Ulcerative } \\
\text { colitis }\end{array}$ & $\begin{array}{l}\text { Crohn's } \\
\text { colitis }\end{array}$ \\
\hline No. of subjects & 35 & 28 & 27 \\
\hline Male/female & $22: 13$ & $14: 14$ & $12: 15$ \\
\hline \multicolumn{4}{|l|}{ Age $(y r)$} \\
\hline Mean & 39.1 & 32.0 & 35.3 \\
\hline Range & $17-94$ & $17-71$ & $21-68$ \\
\hline \multicolumn{4}{|l|}{ Disease duration $(y r)$} \\
\hline Mean & - & 10.1 & 8.0 \\
\hline Range & - & $0.25-30$ & $0.08-21$ \\
\hline \multicolumn{4}{|c|}{ Site of intestinal inflammation } \\
\hline Ileum/colon & 0 & 0 & 10 \\
\hline Total colon & 3 & 12 & 2 \\
\hline Distal colon/rectum & 17 & 16 & 15 \\
\hline Granulomas & 0 & 0 & 11 \\
\hline Strictures/fistulae & 0 & 0 & $5 / 5$ \\
\hline
\end{tabular}

pearance consistent with colitis. More specifically, in those patients with ulcerative colitis, there was absence of fistulization and stricturing. This contrasted with those patients with Crohn's disease of the colon, in whom the presence of strictures, fistulae, segmental involvement, and the presence of granulomas were all documented (Table I).

Histological staining of large bowel mucosal biopsies with fluorescein isothiocyanate (FITC)-conjugated lectins. Large intestinal biopsies were categorized according to their anatomical sites of origin; namely, as either ascending, transverse, descending, or sigmoid colon, and rectum. Biopsies had been previously fixed in formaldehyde and imbedded in paraffin. The retrospectively selected paraffin blocks were then used to prepare sections 4-5 $\mu \mathrm{m}$ thick. Deparaffinization and lectin staining were carried out as previously described (15). In brief, sections were first deparaffinized in xylene before sequential passage through 95,80 , and $70 \%$ ethanols, after which slides were rinsed in $0.01 \mathrm{M}$ phosphatebuffered saline, $\mathrm{pH}$ 7.4. Then, under a dim light, the slides were carefully blotted before treatment with $25 \mu \mathrm{l}(1 \mathrm{mg} / \mathrm{ml})$ of the FITCconjugated lectin. A solution of the same lectin concentration containing $0.15 \mathrm{M}$ sugar hapten to inhibit lectin-binding was applied directly to the surface of adjacent serial tissue sections. After $30 \mathrm{~min}$ in a dark, humid atmosphere, the slides were rinsed with $0.01 \mathrm{M}$ phosphatebuffered saline, $\mathrm{pH} 7.4$, carefully blotted, and then covered with phosphate-buffered glycerol before applying a cover slip. The pattern of fluorescence was then examined with a fluorescence photomicroscope (II; Carl Zeiss, Inc., Thornwood, NY) and photographed for further documentation. Slides were coded so that the observer (P.W.H.) did not know their identity. A number of randomly selected biopsies were restained with FITC-lectins at the end of the study to control for intraobserver variation and to confirm the reproducibility of earlier observations. Morphology was assessed on hematoxylin and eosin stained sections, and the presence of goblet mucin was confirmed with periodic acid Schiff (PAS) as shown in Fig. $1 \mathrm{~A}$. Only those areas containing goblet cells with mucin present were examined. Results were recorded according to the intensity of fluorescence in individual goblet cells. Biopsies were considered to show positive lectin-binding if more than half the total crypts per field demonstrated lectin staining, with a minimum of 10 labeled goblet cells per crypt. Thus, if fluorescence was only present in a patchy distribution, this was classified as 0 . The intensity of individual goblet cell fluorescence was graded using a semiquantitative scale with 0 representing no detectable labeling; $1+$, trace present; $2+$, weak intermediate; $3+$, strong intermediate; $4+$, intense (16). Examples of fluorescein-conjugated lectin-binding to intestinal biopsies are shown in Fig. 1.

Many patients had a series of biopsies taken from multiple sites throughout the large intestine. Some subjects had several biopsies available from the same anatomical region, either taken at the same time or alternatively over a period of several years. Although all available biopsies were subjected to lectin-binding studies, only one biopsy per anatomic region per patient was included in the final subject analysis. The selection of a representative sample was easily determined since the absence or presence of lectin-binding sites was remarkably consistent between multiple biopsies obtained from the same region in the same patient. This was in contrast to the grade of fluorescence intensity, which showed some variation over time, possibly related to disease activity. However, the available clinical data and the limited number of suitable samples did not permit a valid correlation analysis to be undertaken between disease activity and intensity of FITC-lectin fluorescence. The majority of biopsies were obtained in the preceding four years. There was no evidence of a loss of lectin-binding activity as a function of age of the biopsy. The oldest biopsy was $9 \mathrm{yr}$ old and revealed a pattern of lectin-binding similar to that seen with the other biopsies from the same clinical group, some of which were less than 1 yr old. Areas of ulceration and those with loss of mucosal architecture were not included in the final data analysis, since such changes result in a loss of goblet cell number and disruption of normal crypt structure.

Lectins. Plant lectins, purified by affinity chromatography and conjugated with FITC, were obtained from E-Y Laboratories, San 

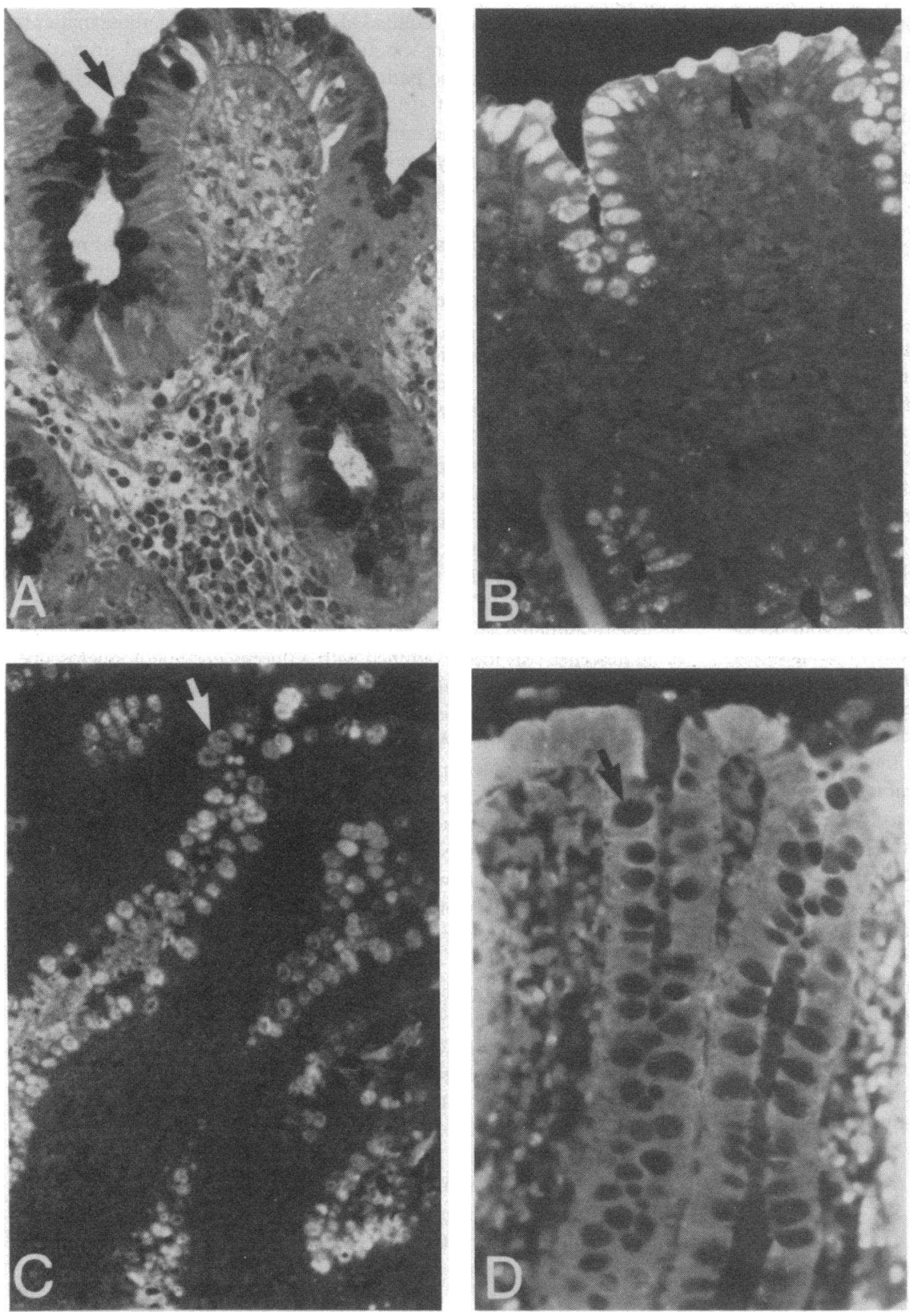

Figure 1. Human colonic mucosal biopsies. $(A)$ Biopsy from a patient with ulcerative colitis, stained with PAS to show the presence of goblet cell mucin (see arrow); $\times 240$. (B) Mucosal biopsy from the colon of a subject with ischemic colitis, stained with FITC-conjugated DBA to show goblet cell mucin (see arrow); $\times 240$. FITC-lectins were incubated with histological sections and examined by fluorescence microscopy as described in Methods.

(C) Biopsy from a patient with ulcerative colitis, stained with FITC-DBA to show the presence of binding sites in goblet cell mucin (see arrow); $\times 240$. (D) Mucosal biopsy from the colon of a subject with Crohn's colitis, stained with FITC-DBA, which did not bind to goblet cell mucin (see arrow). The presence of mucin was confirmed by PAS staining and binding of $\mathrm{RCA}_{1} ; \times 240$.
Mateo, CA. The lectins and their sugar specificities were as follows: $\mathrm{UEA}_{1}$ specifically binds with $\alpha$-L-fucose (Fuc); $\mathrm{RCA}_{1}$, with D-galactose (Gal); and DBA, with $\mathrm{N}$-acetyl galactosamine (GalNAc). The inhibitor carbohydrates Fuc, Gal, and GalNAc (Sigma Chemical Co., St. Louis, MO) were all used at a $0.15-\mathrm{M}$ concentration.

Statistical analyses. The results were analyzed using $\chi^{2}$ and Fisher's Exact Tests (21).

\section{Results}

Although the intensity of fluorescence observed was graded for each slide, such a subjective semiquantitative assessment might not be reproducible by other observers. Therefore, the data were analyzed only to determine the rate of presence of lectin-binding sites. This was achieved by classifying biopsies either as being completely devoid of lectin-binding sites (grade 0 ), or as exhibiting the presence of lectin-binding sites (grades $1+$ or greater). The control group of biopsies was divided into inflamed and noninflamed tissues, according to previous pathological interpretation. The results were expressed as either the number of patients or the number of mucosal biopsies with positive lectin-binding. No statistical differences in the rate of presence of lectin-binding sites between inflamed control patients and those control patients with noninflamed tissues could be demonstrated (results not shown). Because of this, these two subsets were pooled together to form a single control group for further comparison with the tissues from patients with inflammatory bowel disease. Fig. $2 A$ shows the percent of control subjects with lectin-positive biopsies, according to the site of tissue sampling, for the three different lectins used. 


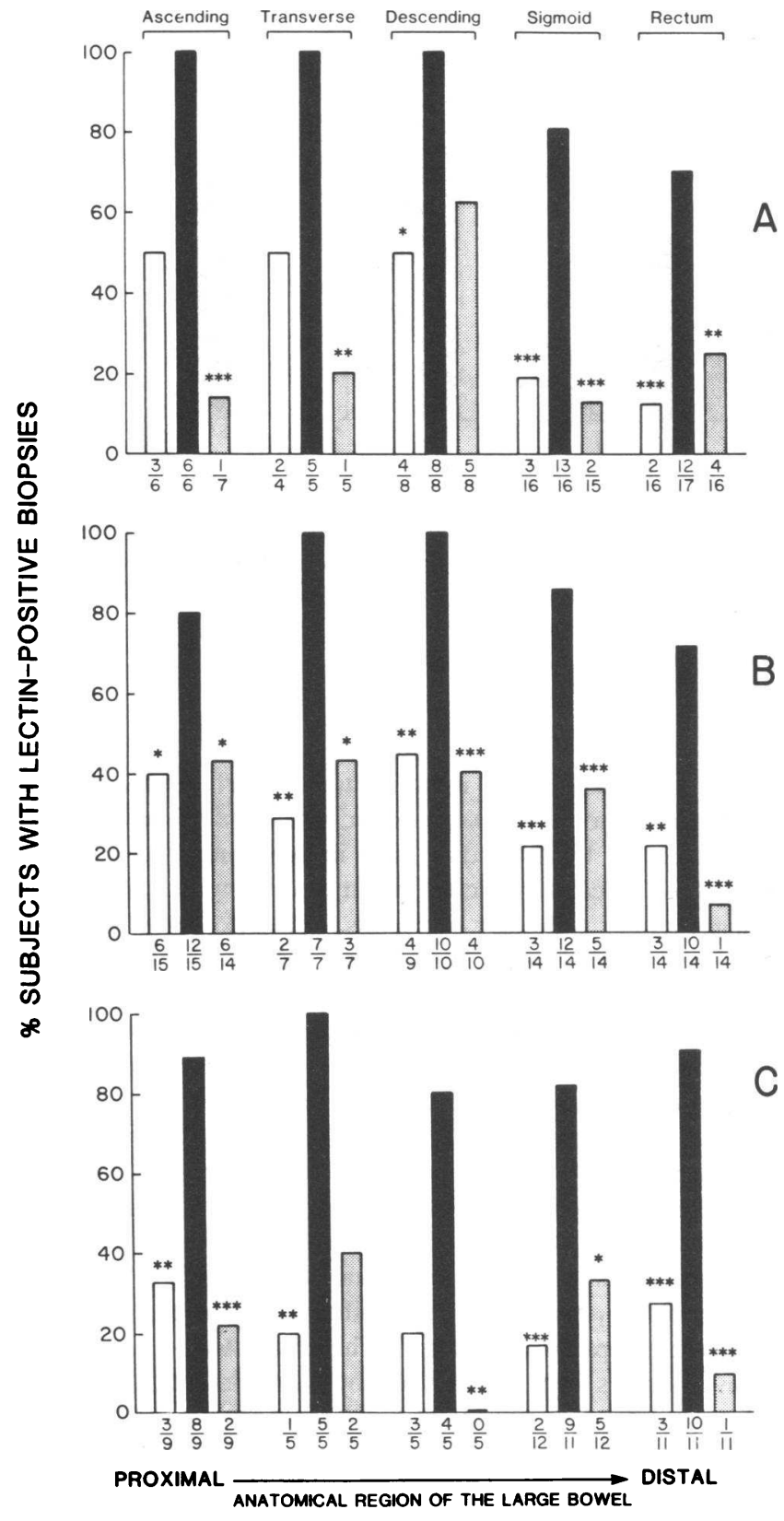

Figure 2. Anatomical distribution of the rate of presence of lectinbinding to colorectal mucin from 35 control subjects and 55 patients with inflammatory bowel disease. $(A) 35$ control subjects, $(B) 28$ patients with ulcerative colitis, and $(C) 27$ patients with Crohn's colitis. Each FITC-lectin was incubated with each biopsy, with and without the specific inhibitor carbohydrate. A positive biopsy was defined as showing a trace or greater fluorescence in a minimum of 10 goblet cells/crypt, with at least half the total crypts/field demonstrating lectin-binding. ${ }^{*} P<0.05 ;{ }^{* *} P<0.025 ;{ }^{* * *} P<0.01-P$ $<0.005$, for $\mathrm{UEA}_{1}$ and DBA groups when compared with $\mathrm{RCA}_{1}$ positive biopsies. $\square, \mathrm{UEA}_{1}$; $\mathbf{n}, \mathrm{RCA}_{1}$; a, DBA.

In the three most proximal regions of the colon, the frequency of $\mathrm{RCA}_{1}$ binding was $100 \%$, dropping to $81.3 \%$ in the sigmoid colon, and $70.6 \%$ in the rectum. With $\mathrm{UEA}_{1}$ (Fig. $2 A$ ), although a similar gradient of binding sites was present, the frequency of staining was much lower, $50 \%$ of controls having available binding sites in the three most proximal portions of the intestine, compared with $18.8 \%$ in the sigmoid, and only $12.5 \%$ in the rectum. In contrast to the regional gradient of lectin-binding sites demonstrated for $\mathrm{RCA}_{1}$ and $\mathrm{UEA}_{1}$ (maximal binding in the proximal colon and minimum binding in the rectosigmoid), the pattern for DBA binding showed no evidence of such a gradient effect. In the proximal colon, the frequency of subjects with positive DBA staining was $<20 \%$, while maximal binding was observed in the descending colon with $62.5 \%$ of subjects showing positive lectin staining, and less than a $25 \%$ positive rate in the rectosigmoid. The frequency of DBA staining was significantly lower than that found with $\mathrm{RCA}_{1}$ in all regions except the descending colon $(P<0.025$ or better), while the frequency of $\mathrm{UEA}_{1}$ staining was lower than that with $\mathrm{RCA}_{1}$ only in the descending colon, sigmoid, and rectum $(P<0.005)$.

A higher frequency of $\mathrm{RCA}_{1}$ binding to colorectal mucin was also observed when ulcerative (Fig. $2 B$ ) and Crohn's colitis (Fig. $2 C$ ) samples were studied. In ulcerative colitis (Fig. $2 \mathrm{~B}$ ), the frequency of $\mathrm{RCA}_{1}$ binding was significantly higher than that of $\mathrm{UEA}_{1}$ or DBA in all five regions of the intestine examined ( $P<0.05$ or better). In Crohn's colitis (Fig. $2 C$ ), the frequency of $\mathrm{RCA}_{1}$ binding to mucin was significantly higher than that of $\mathrm{UEA}_{1}$ in all regions except the descending colon $(P<0.025$ or better), and significantly greater than with DBA $_{1}$ in all regions except the transverse colon $(P<0.05$ or better). In both forms of colitis (Figs. $2 B$ and $C$ ), the existence of a proximal-to-distal gradient in lectin-binding was much less evident than that found for the control group (Fig. $2 \mathrm{~A}$ ).

In all three sets of patients studied (Fig. 2), lectin-binding frequencies in the ascending and transverse regions of the proximal colon were not significantly different. Similarly, the two most distal segments (sigmoid and rectum) revealed comparable patterns and levels of lectin-binding. For these reasons, biopsies from the two most proximal and two most distal segments were pooled and designated as constituting the proximal and distal colon, respectively. Using this anatomical definition, the results for $\mathrm{UEA}_{1}$-binding to control biopsies were further analyzed, as shown in Fig. $3 A$. This confirmed the impression gained from Fig. $2 A$ that there was a significant gradient in the frequency of lectin-binding between the proximal and distal colon. In the proximal colon, $58.3 \%$ of the biopsies were positive, compared with $14.3 \%$ in the distal colon $(P$ $<0.025$ ). Although there was also evidence of a proximal-todistal gradient in ulcerative and Crohn's colitis (Fig. $3 A$ ), this was much less marked than in the control patients, and was not statistically significant. A similar analysis for the results of $\mathrm{RCA}_{1}$ (Fig. $3 \mathrm{~B}$ ) and DBA (Fig. $3 \mathrm{C}$ ) binding demonstrated that lectin-binding in all groups was greater in the proximal than in the distal colon. However, none of these differences was statistically significant.

Because biopsies from the area of the descending colon showed lectin-binding characteristics that were sometimes dissimilar from those observed in the proximal or distal colon (Fig. 2), these data were analyzed separately (Fig. 4). The results showed that with $\mathrm{UEA}_{1}, 50 \%$ of the control subjects demonstrated positive lectin-binding, compared with $44.4 \%$ in the ulcerative colitis, and $20 \%$ in the Crohn's colitis groups. With $\mathrm{RCA}_{1}, 100 \%$ of the control and ulcerative colitis subjects showed positive lectin-binding, compared with $80 \%$ of the Crohn's patients. With DBA, $62.5 \%$ of the control group and $40 \%$ of the ulcerative colitis group showed positive binding, 


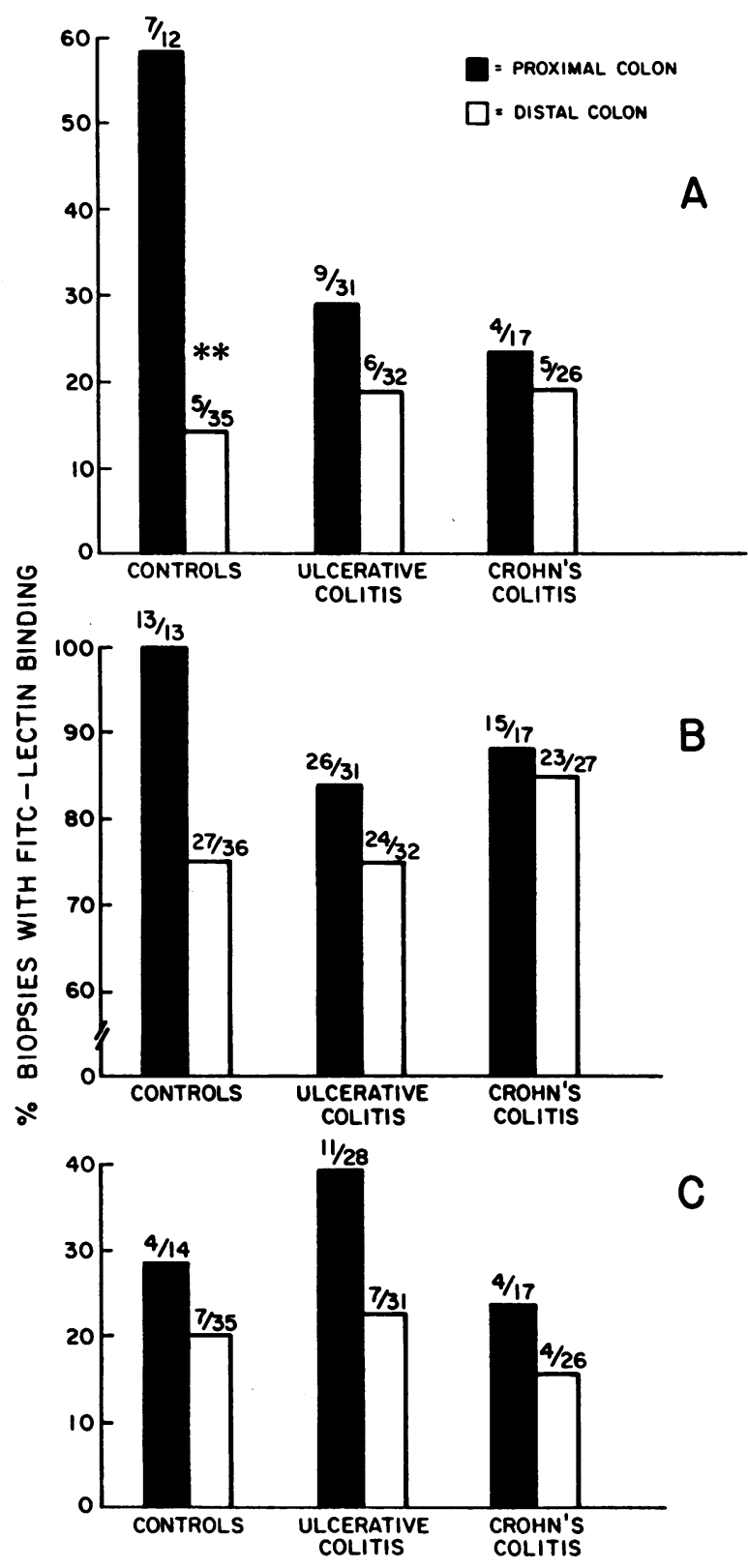

Figure 3. Rate of presence of $(A) \mathrm{UEA}_{1}$ binding sites, $(B) \mathrm{RCA}_{1}$ binding sites, and $(C)$ DBA binding sites in proximal (solid bars) and distal (open bars) colorectal mucin from control subjects and patients with inflammatory bowel disease. Lectin binding and its analysis was performed as described in Fig. 2 and Methods. ${ }^{* *} P<0.025$, proximal compared with distal.

compared with the Crohn's colitis group, in which there was a complete absence of any lectin-binding (Figs. $1 D$ and 4) $(P$ $<0.05$ ). The specificity of this alteration in the Crohn's colitis biopsies was further strengthened by the fact that $80 \%$ of these same tissues demonstrated binding sites for $\mathrm{RCA}_{1}$, and $100 \%$ of the biopsies showed a normal-appearing amount of mucin by PAS staining.

\section{Discussion}

These results provide evidence that the expression of lectinbinding sites in human large intestinal goblet cell mucin is

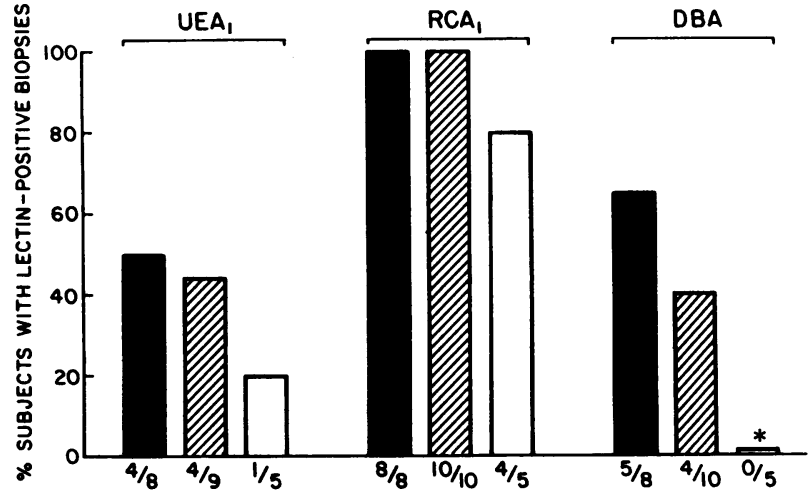

Figure 4. Rate of presence of FITC-lectin binding to descending colonic mucin from controls and patients with inflammatory bowel disease. Binding of $\mathrm{UEA}_{1}, \mathrm{RCA}_{1}$, and DBA to histological sections was carried out as described in Fig. 2 and Methods. ${ }^{*} P<0.05$, for the Crohn's colitis compared with control group. $₫$, controls; ulcerative colitis; $\square$, Crohn's colitis.

altered in patients with inflammatory bowel disease. These changes seem to be disease specific, since analysis of the rate of presence of lectin-binding sites in the control population showed no effect that could be attributed to inflammatory changes alone. Although all lectins tended to show a higher frequency rate of binding to proximal than to distal colorectal mucin, this was only statistically significant for binding to fucosyl residues (UEA $A_{1}$ ) in the control group. The loss of this gradient effect in inflammatory bowel disease seemed to result from a decreased availability of fucosyl residues in the proximal colon of the ulcerative and Crohn's colitis groups (Fig. $3 \mathrm{~A}$ ). Inflammatory bowel disease samples showed additional evidence of a change in colonic mucin differentiation, as measured by the alteration in the relative frequency of lectin-binding sites expressed in the ascending and transverse colon where fucosyl residues (UEA, binding) were significantly reduced relative to galactosyl groups (RCA, binding). This contrasted with proximal colonic samples from the control groups, in which no significant differences between lectin-binding to galactosyl and fucosyl residues could be shown. Ulcerative colitis samples showed uniform changes in every anatomic region from the ascending colon through to the rectum, with higher rates of available galactosyl residues compared with either fucosyl or $\mathrm{N}$-acetyl galactosyl groups. The complete absence of DBA binding to $N$-acetyl D-galactosamine residues in mucin from a small number of descending colon biopsies from Crohn's colitis patients seems to deserve further investigation. Confirming this result in a prospective study, using a larger number of patients, will help determine the value of DBA binding as a sensitive marker for Crohn's colitis. However, the present results indicate that the absence of DBA binding sites was not very specific, since $37.5 \%$ of controls and $60 \%$ of ulcerative colitis patients also demonstrated absence of DBA binding in the descending colon. Unpublished observations from this laboratory on several patients with Crohn's Disease, limited to the small bowel, indicate that GalNAc residues continue to be expressed in the descending colon. Because of the retrospective design of this study, it was not possible to reliably correlate disease activity with lectin binding. However, based on these 
preliminary findings, it seems worthwhile to look for evidence of such a relationship using a prospectively designed experiment.

The effects of mucosal inflammation on colorectal mucin lectin-binding sites has not to our knowledge previously been investigated. Using cytochemical techniques, Ehsanullah et al. (3) studied mucin chemistry in nonspecific proctitis, and as with the present study, found no change from normal patients, in contrast to the abnormal patterns seen in Crohn's Disease and ulcerative colitis. Yonezawa et al. $(17,19)$ examined the nonneoplastic mucosa of patients with colorectal tumors and found that with right-sided tumors, all patients have demonstrable UEA $_{1}$-binding sites in the right colon, whereas with left-sided tumors, the distal nonneoplastic mucosa contained binding sites in only $10.3 \%$ of cases, while in patients with rectal carcinoma, the nonneoplastic mucosa was completely devoid of $\mathrm{UEA}_{1}$-binding sites. In comparison, our own results for $U_{E A}$ binding show far less specificity. This is probably due to differences in clinical material, patients with any history or evidence of colorectal neoplasia having been excluded from our control group, whereas the control biopsies in the study of Yonezawa et al. were taken from the same subjects with colorectal cancer. Similarly, the present results cannot be validly compared with those of Boland et al. (16), who obtained their normal tissues predominantly from patients with colon cancer. These authors (16) did not analyze their results according to anatomic region, nor did they estimate the rate of presence of lectin-binding sites. Control groups of this sort $(16,17,19)$ can no longer be regarded as reliably representing normal mucosa, since it has now been well established that even the histologically normal mucosa of human large bowel bearing carcinomas frequently shows abnormal mucin composition $(20,22,23)$. It has further been suggested that such changes in mucin glycoprotein synthesis antedate malignant changes and may serve as markers for premalignant epithelium $(20,22,23)$. The variations in mucin composition that have been reported in ulcerative colitis associated with carcinoma $(24,25)$ and dysplasia (3) are not present when the disease is in remission, and are not found in areas of reparative hyperplasia $(24,25)$. Note that none of the ulcerative colitis patients in the present study had any history or evidence of colorectal cancer.

It is generally accepted that there is a sequential scheme for goblet cell mucin glycosylation, in which carbohydrate residues such as Fuc, Gal, and GalNAc may be added terminally (26). However, expression of these terminal carbohydrate residues may be further modified by the enteric microflora, which have been shown to extensively degrade mucin carbohydrate moieties (27). Thus, the final availability of lectinbinding sites in human colorectal mucin seems to be determined not only by biosynthetic factors, but also by those that produce degradation. As a result of the more rapid rate of cell turnover in inflammatory bowel disease $(6,7)$, less time may be available to allow full differentiation of mucin, thus resulting in a relative reduction in certain terminal carbohydrate residues such as fucose. This might also explain the decreased rate of presence of $\mathrm{UEA}_{1}$ binding sites in the proximal colon of Crohn's and ulcerative colitis, resulting in a loss of the normal proximal-to-distal gradient of mucin fucosyl expression.

The results of the present study add further weight to the growing body of evidence that indicates that there are specific biochemical alterations in the colorectal mucin of patients with Crohn's and ulcerative colitis. Whether these changes are the basis or simply just another manifestation of the disease process still remains to be determined.

\section{Acknowledgments}

The authors wish to thank Dr. Burton Goldfine, James Hansen, and Dr. David Watson for providing clinical material.

This study was supported in part by a Student Fellowship from the American Gastroenterological Association, awarded to Dr. Huber, and by grants from the National Foundation for Ileitis and Colitis, Inc., the School of Medicine, University of California, Davis, and the Cancer Research Coordinating Committee, University of California.

\section{References}

1. Filipe, M. I. 1969. Value of histochemical reactions for mucosubstances in the diagnosis of certain pathological conditions of the colon and rectum. Gut. 10:577-586.

2. Filipe, M. I., and I. Dawson. 1970. The diagnostic value of mucosubstances in rectal biopsies from patients with ulcerative colitis and Crohn's Disease. Gut. 11:229-234.

3. Ehsanullah, M., M. I. Filipe, and B. Gazzard. 1982. Mucin secretion in inflammatory bowel disease: correlation with disease activity and dysplasia. Gut. 23:485-489.

4. Gad, A. 1969. A histochemical study of human alimentary tract mucosubstances in health and disease. II. Inflammatory conditions. Br. J. Cancer. 23:64-68.

5. Greco, V., G. Lauro, A. Fabrini, and T. Torsoli. 1967. Histochemistry of the colonic epithelial mucins in normal subjects and in patients with ulcerative colitis. Gut. 8:491-496.

6. Eastwood, G. L., and J. S. Trier. 1973. Epithelial cell renewal in cultured rectal biopsies in ulcerative colitis. Gastroenterology. 64:383390.

7. Serafini, E. P., A. P. Kirkwood, and T. J. Chambers. 1981. Rate and pattern of epithelial cell proliferation in ulcerative colitis. Gut. 22:648-652.

8. Clamp, J. R., A. Allen, R. A. Gibbons, and G. P. Roberts. 1978 Chemical aspects of mucus. Br. Med. Bull. 34:25-41.

9. Kim, Y. S., and R. Isaacs. 1979. Glycoprotein metabolism in inflammatory and neoplastic diseases of the human colon. Cancer Res. 35:2092-2097.

10. Goodman, M. J., P. W. Kent, and S. C. Truelove. 1977. Glucosamine synthetase activity of the colonic mucosa in ulcerative colitis and Crohn's disease. Gut. 18:219-228.

11. Clamp, J. R., G. Fraser, and A. E. Read. 1981. Study of the carbohydrate content of mucus glycoproteins from normal and diseased colons. Clin. Sci. (Lond.). 61:229-234.

12. Teague, R. H., D. Fraser, and J. R. Clamp. 1973. Changes in monosaccharide content of mucus glycoproteins in ulcerative colitis. Br. Med. J. 2:645-646.

13. Podolsky, D. K., and K. J. Isselbacher. 1983. Composition of human colonic mucin: selective alteration in inflammatory bowel disease. J. Clin. Invest. 72:142-153.

14. Etzler, M. E. 1979. Lectins as probes in studies of intestinal glycoproteins and glycolipids. Am. J. Clin. Nutr. 32:133-138.

15. Jacobs, L. R., D. De Fontes, and K. L. Cox. 1983. Cytochemical localization of small intestinal glycoconjugates by lectin histochemistry in controls and subjects with cystic fibrosis. Dig. Dis. Sci. 28:422-428.

16. Boland, C. R., C. K. Montgomery, and Y. S. Kim. 1982. Alterations in human colonic mucin occurring with cellular differen- 
tiation and malignant transformation. Proc. Natl. Acad. Sci. USA. 79:2051-2055.

17. Yonezawa, S., T. Nakamura, S. Tanaka, and E. Sato. 1982. Glycoconjugate with Ulex europaeus agglutinin-1-binding sites in normal mucosa, adenoma and carcinoma of the human large bowel. J. Natl. Cancer Inst. 69:777-781.

18. Boland, C. R., P. Lance, B. Levin, R. H. Riddell, and Y. S. Kim. 1982. Lectin binding indicates an abnormality of the goblet cell glycoconjugates in ulcerative colitis. Gastroenterology. 82:1021a. (Abstr.).

19. Yonezawa, S., T. Nakamura, S. Tanaka, K. Marita, M. Nishi, and E. Sata. 1983. Binding of Ulex europaeus agglutinin-1 in polyposis coli: comparative study with solitary adenoma in the sigmoid colon and rectum. J. Natl. Cancer Inst. 71:19-24.

20. Filipe, M. I., S. Mughal, and H. J. Bussey. 1980. Patterns of mucus secretion in the colonic epithelium in familial polyposis. Invest. Cell. Pathol. 3:329-343.
21. Steele, R. G. D., and J. H. Torrie. 1980. Principles and Procedures of Statistics. Second ed. McGraw-Hill Inc., New York.

22. Dawson, P. A., J. Patel, and M. I. Filipe. 1978. Variations in sialomucins in the mucosa of the large intestine in malignancy. A quantimet and statistical analysis. Histochem. J. 10:559-572.

23. Rogers, C. M., K. B. Cooke, and M. I. Filipe. 1978. Sialic acids of human large bowel mucosa: O-acylated variants in normal and malignant states. Gut. 19:587-592.

24. Filipe, M. I. 1977. Mucin histochemistry in the detection of early malignancy in the colonic epithelium. Adv. Exp. Med. Biol. $89: 413-422$.

25. Filipe, M. I. 1979. Mucins in the gastrointestinal epithelium. A review. Invest. Cell. Pathol. 2:195-216.

26. Phelps, C. F. 1978. Biosynthesis of mucus glycoprotein. Br. Med. Bull. 34:43-48.

27. Variyam, E. P., and L. C. Hoskins. 1981. Mucin degradation in human colon ecosystems. Degradation of hog gastric mucin by cecal extracts and fecal cultures. Gastroenterology. 81:751-758. 